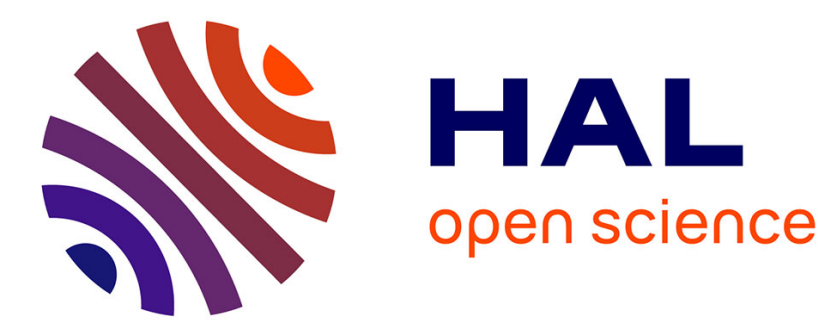

\title{
Analysis of the IEEE 802.11 EDCF scheme for broadcast traffic: Application for VANETs
}

Younes Bouchaala, Paul Mühlethaler, Nadjib Achir

\section{To cite this version:}

Younes Bouchaala, Paul Mühlethaler, Nadjib Achir. Analysis of the IEEE 802.11 EDCF scheme for broadcast traffic: Application for VANETs. 2017 Wireless Days, Mar 2017, Porto, Portugal. pp.252 257, 10.1109/WD.2017.7918156 . hal-01617895

\section{HAL Id: hal-01617895 \\ https://hal.science/hal-01617895}

Submitted on 17 Oct 2017

HAL is a multi-disciplinary open access archive for the deposit and dissemination of scientific research documents, whether they are published or not. The documents may come from teaching and research institutions in France or abroad, or from public or private research centers.
L'archive ouverte pluridisciplinaire HAL, est destinée au dépôt et à la diffusion de documents scientifiques de niveau recherche, publiés ou non, émanant des établissements d'enseignement et de recherche français ou étrangers, des laboratoires publics ou privés. 


\title{
Analysis of the IEEE 802.11 EDCF scheme for broadcast traffic: Application for VANETs
}

\author{
Younes Bouchaala ${ }^{\dagger}$, Paul Muhlethaler*, and Nadjib Achir ${ }^{\ddagger}$ \\ *INRIA EVA, Centre de Recherche de Paris, 2 Rue Simone, IFF CS 42112, 75589 Paris Cedex 12 \\ Email: \{paul.muhlethaler\}@inria.fr \\ †Institut VEDECOM, 77, rue des Chantiers, 78000 Versailles, France \\ Emails: \{younes.bouchaala, oyunchimeg.shagdar\}@ vedecom.fr \\ $\ddagger$ Universite Paris 13, Sorbonne Paris Cite - L2TI, 99 Avenue J-B Clément, 93430 Villetaneuse, France \\ Email: nadjib.achir@univ-paris13.fr
}

\begin{abstract}
In this paper we propose a model of the IEEE 802.11 Enhanced Distributed Coordination Function (EDCF) which can build different access priorities for different classes of traffic. These priorities are obtained using different inter frame spacings called Arbitration Inter Frame Spacings (AIFSs) to differentiate the access of different classes of traffic. When a node has a pending packet it must first wait for the channel to become idle for a given number $A$ of mini-slots $\sigma$ before starting to decrement its back-off; this interval of $A$ mini-slots $\sigma$ is called the AIFS. If the channel becomes busy before this back-off expires, then the node will have to to wait for another $A$ mini-slots before starting to decrement its back-off again. The nodes can also use different back-off windows to further differentiate between different classes of traffic. The model we propose is much simpler than previous models [1], [2], [3] and handles the more general case of a Poisson arrival for the traffic. Moreover, the model presented here is designed for broadcast traffic whereas most models handle pointto-point IEEE 802.11 transmission.
\end{abstract}

When we have two classes of traffic, the model leads to two coupled non-linear equations involving the transmission rates in each class of traffics. These equations can be easily solved using simple numerical methods. The model then allows the successful rate or the throughput for each class of traffic to be computed in a straightforward manner. Numerical examples derived from VANET scenarios show the direct and positive influence of the Arbitration Inter Frame Spacings on the performance of each class of traffic.

Keywords-CSMA, EDCF, Markov Chains, Steady states .

\section{INTRODUCTION}

Wireless Access in Vehicular Environments (WAVE) [4]. uses the IEEE $802.11 \mathrm{p}$ as an access scheme to share the radio medium dedicated to Vehicular Ad Hoc Neworks (VANETs). IEEE802.11p is designed to provide communication between vehicles $(\mathrm{V} 2 \mathrm{~V})$, and between vehicles and roadside infrastructure (V2I) in new Intelligent Transportation Systems (ITS). One of the prominent applications in V2V communication concerns safety applications; these applications relies on the periodic transmission of packets. There are two main types of applications Car Awareness Messages (CAM) [5] and Decentralized Environmental Notification Messages (DENM) [6]. Car Awareness Messages are used to periodically send information about the vehicles' velocities and positions while DENMs are sent when a hazardous event occurs on the road.
Since IEEE 802.11 is inherently a random access protocol the medium is shared by all the vehicles which compete to transmit their packets. This can create a problem since safety messages may carry traffic of vital information with stringent deadline for their actual delivery. For instance, CAMs or DENMs sent just after a hazardous event carry very urgent information whose delivery might be vital for neighboring vehicles. In the amendment IEEE 802.11e, the IEEE 802.11 group has defined a special mechanism to create different type of access. This mechanism, called the Enhanced Distributed Coordination Function (EDCF) mainly relies on different inter frame spacing called AIFSs for Arbitration Inter Frame Spacings. In this paper we propose a simple model to analyze EDCF for broadcast packets i.e. without acknowledgment and potential retransmission and with a Poisson packet arrival model. We show that the influence of different AIFSs is huge on performance for different classes of traffic.

The remainder of this paper is organized as follows: Section II briefly reviews related work; Section III describes the model proposed to study EDCF. This model is based on coupled and simultaneous Markov chains for which we compute the steady state. We obtain the steady-state transmission rate of each class of traffic. The computation of the throughput of each class of traffic also requires studying the evolution of the position of in Section IV we compute the percentage of successful transmissions and the throughput for each class of traffic. Finally, Section V concludes the paper.

\section{RELATED WORK}

The seminal paper by Kleinrock [7] gave rise to early studies of CSMA, all of which highlighted the improvement carrier sensing made to Aloha, which was the standard protocol at the time. None of these studies, however, provided a satisfactory model of the back-off techniques that CSMA systems used.

Such a model was, however, provided by Bianchi [8], which led to a breakthrough in the analysis of the backofff techniques used in protocols such as IEEE 802.11.

In recent studies, the Enhanced Distributed Coordination Function was analyzed with techniques stemming from those employed by Bianchi. However these studies [1], [2], [3] focus on point-to-point transmission with a saturated network. Moreover, they use complex Markov chains with a large number 
of states. In contrast our study deals with the more general Poisson packet arrival model and also exhibits a Markov chain with a reasonable number of states. We obtain a simple and closed formula for the busy rate ratio.

It must be noted that in this paper we consider that all the nodes are within carriers sense range and thus we do not study the spatial effect of EDCF; the spatial effect of CSMA has been studied in [9] [10] [11].

\section{SYSTEM MODEL}

The model uses the following assumption for the IEEE 802.11 protocol: the packet length is $T$ and the length of the minislot for sensing is denoted by $\sigma$. Rather than studying pointto-point transmissions we focus on transmission of broadcast packets thus without acknowledgment. Moreover, the model for the arrival is a Poisson model with a single buffer with loss and an arrival rate $\lambda$. We assume that the number of stations $M$ sharing the same radio spectrum is known.

The IEEE $802.11 \mathrm{p}$ protocol with priority uses different inter frame spacings called AIFSs for Arbitration Inter Frame Spacings to differentiate various classes of traffic. When a node has a pending packet it must first wait for the channel to become idle for a given number $A$ of mini-slots $\sigma$ before starting to decrement its back-off; this interval of $A$ mini-slots $\sigma$ is called the AIFS, see Figure III.1. If the channel becomes busy before this back-off expires, then the node will have to wait for another $A$ mini-slots before starting to decrement its back-off again.

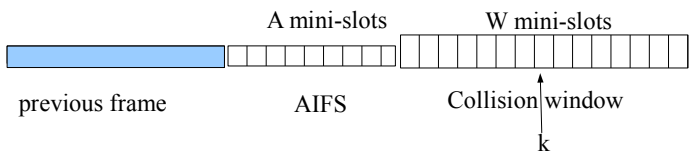

A node must wait A mini-slots and a random back-of of $\mathrm{k}$ slots $0 \leq \mathrm{k} \leq \mathrm{W}-1$.

If a transmission occurs, the node must wait the A mini-slots again.

Fig. III.1. Back-off procedure with IEEE 802.11 EDCF scheme.

We first study the steady state of an IEEE 802.11p network which includes the AIFS mechanism.

\section{A. Steady-state of an IEEE 802.11p with AIFS}

The state diagram of IEEE 802.11p with priorities is shown in Figure III.2.

The state at the top of the diagram represents the probability that the node is idle i.e. without any packet waiting for transmission and we call $c_{I}$ the probability of the node being in this state at equilibrium.

The $A$ states just below represent the states when the node starts waiting for the AIFS. We denote by $c_{i}$ for $1 \leqslant i \leqslant A$ the probability that the node has waited for $i$ idle slots in the AIFS part of the waiting time before beginning its transmission.

The $W$ states represented horizontally correspond to the backoff states of the node. The state $k$ with $0 \leqslant k \leqslant W-1$ represents the state that the node, having already waited for the entire AIFS interval is now in back-off and still has $i$ minislots of back-off to wait. We call $b_{i}$ for $0 \leqslant k \leqslant W-1$ the probability that the node still has $k$ mini-slots to wait before it can access the channel. The states $(j, i) 0 \leqslant j \leqslant W-$ $10 \leqslant i \leqslant A$ illustrated vertically correspond to the case when a back-off procedure (at stage $j$ ) has been interrupted by a transmission and the node has to wait $A$ mini-slots again.

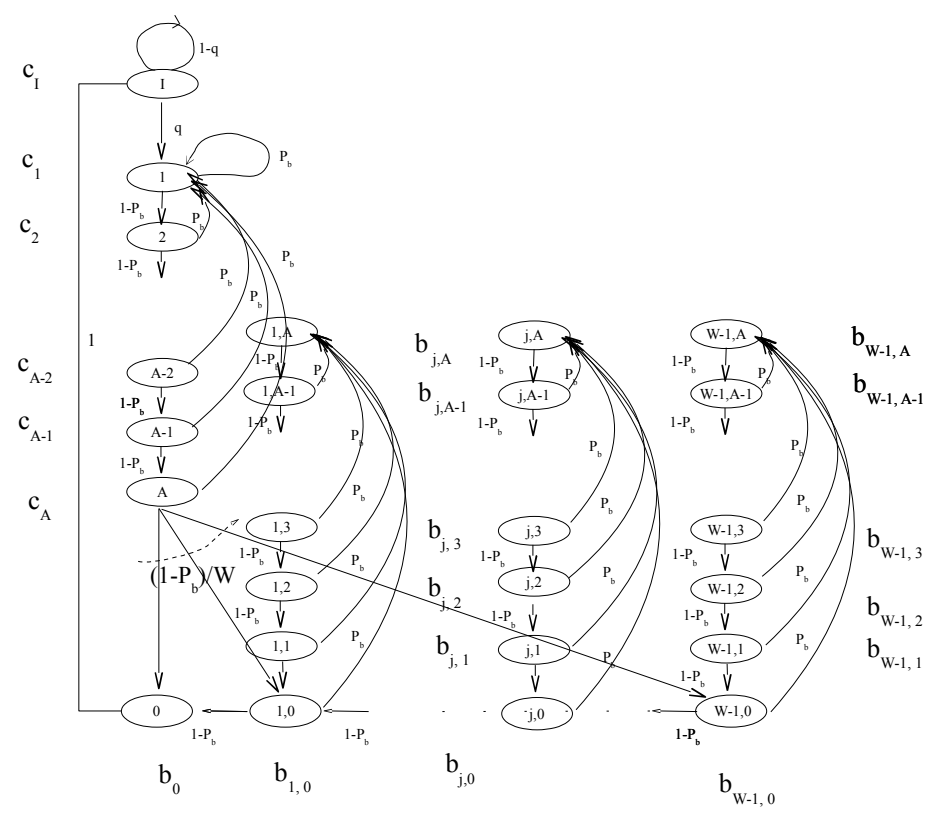

Fig. III.2. State diagram of the back-off scheme with retransmission with AIFS. $A$ contiguous mini-slots must be idle before a transmission can be made and a transmission in one these mini-slots re-start the whole process.

The transitions are

- when a packet arrives at an idle node, the node starts to wait for the AIFS,

- $\quad$ in the AIFS states, the number of idle slots already sensed increases when the current mini-slot on the channel is idle (with probability $1-P_{b}$ ) and the AIFS count down must begin again if the transmission of a packet starts in the current mini-slot (with probability $\left.P_{b}\right)$,

- $\quad$ in the back-off states, the remaining number of minislots that a node must still wait decreases when the current mini-slot on the channel is idle (with probability $1-P_{b}$ ) and the AIFS count down begins again if the transmission of a packet starts in the current mini-slot (with probability $P_{b}$ ),

- when the back-off reaches 0 , the pending packet is transmitted and the state of the node becomes the idle state.

We can see all these transitions in Figure III.2. $c_{I}, c_{1}, c_{2}, \ldots, c_{A}, b_{0}, b_{i, j} 1 \leqslant i \leqslant A, 1 \leqslant j \leqslant W-1$ are the steady-state probabilities of the corresponding state. The transitions are shown by arrows and the transition probabilities are indicated, see Figure III.2.

We analyze the probability flow between state $I$ (probability $c_{I}$ ) and state $(0,0)$ (probability $\left.b_{0}\right)$. The equation of the steady state is :

$$
(1-q) c_{I}+b_{0}=c_{I}
$$


which is equivalent to

$$
q c_{I}=b_{0}
$$

where $q$ denotes the probability that at least one packet arrives at a node during the mean duration during two observation instants of the Markov chain. The instants at which we observe our Markov chain are at the end of the sensing slots (slots of duration $\sigma$ ) if the channel is empty or after the transmission of a packet (of duration $T$ ) if there is a transmission. Thus the mean duration between two instants of observation is $(1-$ $\left.P_{b}\right) \sigma+P_{b} T$ and

$$
q=1-e^{-\lambda\left(\left(1-P_{b}\right) \sigma+P_{b} T\right)} .
$$

Writing the equation for the states $i$ for $1 \leqslant i \leqslant A$, we obtain:

$$
c_{i}=\left(1-P_{b}\right)^{i-1} c_{1} \text { for } 1 \leqslant i \leqslant A .
$$

Concerning the states $(W-1, A),(W-1, A-1), \ldots,(W-$ $1,1),(W-1,0)$ we have the following equations :

$$
b_{W-1, A-i}=\left(1-P_{b}\right)^{i} b_{W-1, A} \quad 1 \leqslant i \leqslant A,
$$

and

$$
\begin{gathered}
b_{W-1, A}=P_{b}\left(b_{W-1, A}+b_{W-1, A-1} \ldots b_{W-1,0}\right), \\
b_{W-1,0}=\left(1-P_{b}\right) b_{W-1,1}+\frac{c_{A}\left(1-P_{b}\right)}{W} .
\end{gathered}
$$

Solving these equations, we find:

$$
b_{W-1, i}=\frac{P_{b}}{\left(1-P_{b}\right)^{i}} \frac{c_{A}}{W} \quad 1 \leqslant i \leqslant A,
$$

and

$$
b_{W-1,0}=\frac{c_{A}}{W}
$$

Similarly for states $(j, A),(j, A-1), \ldots,(j, 1),(j, 0)$ we obtain:

$$
\begin{gathered}
b_{W-j, i}=\frac{P_{b}}{\left(1-P_{b}\right)^{i}} \frac{j c_{A}}{W} 1 \leqslant i \leqslant A, \quad 1 \leqslant j \leqslant W-1, \\
b_{W-j, 0}=\frac{j c_{A}}{W} 1 \leqslant j \leqslant W-1 .
\end{gathered}
$$

We can now compute

$$
\sum_{i=0}^{A} b_{j, i}=\frac{1}{\left(1-P_{b}\right)^{A}} \frac{j c_{A}}{W} \quad 1 \leqslant j \leqslant W-1
$$

and thus:

$$
\begin{gathered}
\sum_{j=1}^{W-1} \sum_{i=0}^{A} b_{j, i}=\frac{c_{A}}{\left(1-P_{b}\right)^{A}} \frac{W-1}{2} . \\
b_{0}=\frac{W-1}{W} c_{A}\left(1-P_{b}\right)+\left(1-P_{b}\right) \frac{c_{A}}{W}=\left(1-P_{b}\right) c_{A}
\end{gathered}
$$

$$
b_{0}=\left(1-P_{b}\right)^{A} c_{1}
$$

The normalization condition is :

$$
\sum_{j=1}^{W-1} \sum_{i=0}^{A} b_{j, i}+b_{0}+\sum_{i=1}^{A} c_{i}+c_{I}=1
$$

We obtain :

$$
c_{1}\left(\frac{W-1}{2\left(1-P_{b}\right)}+\left(1-P_{b}\right)^{A}\left(1+\frac{1}{q}\right)+\frac{1-\left(1-P_{b}\right)^{A}}{P_{b}}\right)=1
$$

and thus :

$$
b_{0}=\frac{\left(1-P_{b}\right)^{A}}{\left(\frac{W-1}{2\left(1-P_{b}\right)}+\left(1-P_{b}\right)^{A}\left(1+\frac{1}{q}\right)+\frac{1-\left(1-P_{b}\right)^{A}}{P_{b}}\right)}
$$

This equation is a fixed-point equation in $b_{0}$ because $P_{b}=$ $1-\left(1-b_{0}\right)^{M-1}$ when there is only one type of node i.e. the same $A$ and $W$. To check this equation, we observe that when $A=0$ we obtain the formula which is in [12].

In the next section, we will study two interdependent Markov chains corresponding to coexisting nodes which have different values of $A$.We now focus on the analysis of a network containing two kinds of nodes that have different AIFSs and operate with different values of $A$.

\section{B. Steady-state of the mini-slots observed after the AIFS}

We propose to combine nodes using different AIFSs. Although it is possible to build a model with more than two AIFSs, we will present the computation for only two different AIFSs. We consider the traffic with the highest priority with an AIFS $A_{1}$ and a collision window $W_{1}$ and the low priority traffic with an AIFS $A_{2}>A_{1}$ and a collision window $W_{2}$. We set $L_{1}=A_{2}-A_{1}$ and $L_{2}=\min \left(W_{1}, W_{2}\right)-L_{1}$.

In Figure III. 3 we show the state producing $i$ idle mini-slots after an AIFS of $A_{1}$ mini-slots. We have a transition rate of $1-p_{b}$ from state $i$ to state $i+1$ for $i \leqslant L_{1}$ and a transition rate of $1-p_{b}^{\prime}$ from state $i$ to state $i+1$ for $0 \leqslant L_{1} \leqslant i \leqslant L_{1}+L_{2}$. $p_{b}$ corresponds to the probability of a busy slot when only the high priority traffic can be sent and $p_{b}^{\prime}$ (with $p_{b}^{\prime}>p_{b}$ corresponds to the probability of the current mini-slot being busy when both high and low priority traffic can be sent.

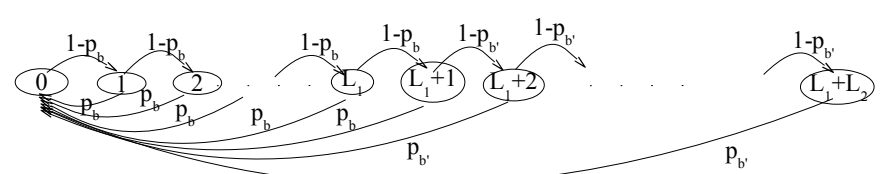

Fig. III.3. State diagram of the state of the channel after the end of a transmission and $A_{1}$ mini-slots.

Let $d_{i}$ with $0 \leqslant i \leqslant L_{1}+L_{2}$ be the steady-state probability of state $i$. We have:

$$
d_{i}=\left(1-p_{b}\right)^{i} d_{0} \text { with } 0 \leqslant i \leqslant L_{1}+1,
$$


$d_{i}=\left(1-p_{b}\right)^{L_{1}+1}\left(1-p_{b}^{\prime}\right)^{i-L_{i}+1} d_{0}, L_{1}+2 \leqslant i \leqslant L_{1}+L_{2}+1$,

and we complete the computation using the normalization rule. Thus the probability of being in the first part of the back-off window ( $L_{1}$ first mini-slots) is:

$$
\begin{aligned}
& p_{1}=\frac{1-\left(1-p_{b}\right)^{L_{1}+1}}{p_{b}}\left(\frac{1-\left(1-p_{b}\right)^{L_{1}+1}}{p_{b}}\right. \\
&\left.+\left(1-p_{b}\right)^{L_{1}+1} \frac{1-\left(1-p_{b}^{\prime}\right)^{L_{2}+1}}{p_{b}^{\prime}}\right)^{-1} .
\end{aligned}
$$

The probability of being in the second part of the back-off windows ( $L_{2}$ last mini-slots) is:

$$
\begin{aligned}
p_{2}=\left(1-p_{b}\right)^{L_{1}+1} & \frac{1-\left(1-p_{b}^{\prime}\right)^{L_{2}+1}}{p_{b}^{\prime}}\left(\frac{1-\left(1-p_{b}\right)^{L_{1}+1}}{p_{b}}\right. \\
& \left.+\left(1-p_{b}\right)^{L_{1}+1} \frac{1-\left(1-p_{b}^{\prime}\right)^{L_{2}+1}}{p_{b}^{\prime}}\right)^{-1}
\end{aligned}
$$

$p_{1}$ and $p_{2}$ can be used to weight the probabilities of the different back-off situations as shown in the next section.

\section{Performance of the system with two classes of traffic using different AIFSs}

We assume that we have two IEEE 802.11p networks sharing the radio spectrum and using different value of $A$ (respectively $A_{1}, A_{2}$ ) with $M_{1}$ nodes in the first network and $M_{2}$ nodes in the second network.

For the first type of nodes we call $\tau_{1}$ the transmission rate in the steady state. For the second type of nodes we call $\tau_{2}$ the transmission rate in the steady state. Thus we have Equation III.1 with $b_{0}=\tau_{1}, P_{b}=1-\left(1-\tau_{1}\right)^{M_{1}-1}\left(1-\tau_{2}\right)^{M_{2}-1}$, with $A=A_{1}$ for the first type of nodes. We have Equation III.1 with $b_{0}=\tau_{2}, P_{b}=1-\left(1-\tau_{1}\right)^{M_{1}-1}\left(1-\tau_{2}\right)^{M_{2}-1}$ and $A=A_{2}$ for the second type of nodes. Thus we have two nonlinear and coupled equations in $\tau_{1}$ and $\tau_{2}$ that must be solved numerically. We can use Maple to perform this task.

We now use the analysis developed in Subsection III-B. The first part of the back-off window with only the high traffic contains $L_{1}=A_{2}-A_{1}$ mini-slots and we still have $L_{2}=$ $W-\left(A_{2}-A_{1}\right)$ mini-slots to complete the back-off window. We are not taking into account the last mini-slots of the backoff window where there are only arrivals of low priority traffic.

We also have:

$p_{b}=1-\left(1-\tau_{1}\right)^{M_{1}-1}, p_{b}^{\prime}=1-\left(1-\tau_{1}\right)^{M_{1}-1}\left(1-\tau_{2}\right)^{M_{2}-1}=P_{b}$.

We can compute the success rate for a node belonging to the first kind of nodes. In the first part of the back-off window the transmission is successful when exactly one high priority node transmits. In the second part of the back-off the transmission is successful when exactly one high priority node transmits and no low priority node transmits. Thus the success rate for the high priority nodes can be written as succ $_{1}=p_{1} M_{1} \tau_{1}\left(1-\tau_{1}\right)^{M_{1}-1}+p_{2} M_{1} \tau_{1}\left(1-\tau_{1}\right)^{M_{1}-1}\left(1-\tau_{2}\right)^{M_{2}-1}$

For the low priority nodes the success rate is simply:

$$
\operatorname{succ}_{2}=M_{2} \tau_{2}\left(1-\tau_{2}\right)^{M_{2}-1}\left(1-\tau_{1}\right)^{M_{1}}
$$

The normalized throughput for the high priority nodes is :

$$
t h r_{1}=\frac{\operatorname{succ}_{1} T}{P_{b} T+\sigma\left(1-P_{b}\right)}
$$

The normalized throughput for the low priority nodes is :

$$
t h r_{2}=\frac{\operatorname{succ}_{2} T}{P_{b} T+\sigma\left(1-P_{b}\right)}
$$

The formulas obtained here can be generalized with more than two classes of traffic.

\section{Performance of the system with two classes of traffic using different collision windows}

If we study two classes of coexisting nodes using EDCA with the same value of $A$ but different values for the $W$ (e.g $W_{1}$ and $W_{2}$ ) we will obtain two coupled equations of type III.1 in $\tau_{1}$ and $\tau_{2}$ with $W_{1}$ and $W_{2}$. These equations can be easily solved numerically. Since the value of $A$ is the same in the two kinds of nodes the formulas derived in Subsection III-B are no longer useful and a direct computation of the successful probability and the normalized throughput can be computed for both kinds of traffic.

\section{RESULTS OF THE MODEL FOR THE IEEE 802.11 WITH PRIORITIES}

We use the previous model to compute the effect of the AIFS with two populations of $M$ vehicles and we use the same figures i.e. packet inter-arrival in a node: $100 \mathrm{~ms}, \sigma=77$ bits. The packet size, including the overhead, is $T=3998$ bits and the data rate is $6 \mathrm{Mbps}$ thus $\lambda=1 / 600000$. We use (if not otherwise specified) $l=25 \mathrm{~m}$ for the mean distance between two vehicles in a lane and we vary the carrier sense range $\left(C_{c s}\right.$ in meter) to modify the number $M$ of contending vehicles. We assume that all the vehicles within the carrier sense range share the same medium and can collide. Thus $M=\frac{2 n b \times C_{c s}}{l}$ where $n b$ is the number of lanes, here we assume $n b=2$.

The first type of the $M_{1}$ vehicles uses an IEEE 802.11 access with an AIFS with $A_{1}=1$ slots and $W=32$. The second type of the $M_{2}$ vehicles uses an IEEE 802.11 access with an AIFS with $A_{2}=6$ slots and $W=32$. We have $M_{1}+M_{2}$ vehicles contending for access. We thus have $L_{1}=5$ and $L_{2}=27$.

In Figure IV.1, we show the probability of success for the two classes of traffic which, is the probability that a transmitted packet is actually received. When the carrier sense range increases, more vehicles are in the same contention area and thus the probability of success tends to decrease. We observe that the EDCF scheme with $A=1$ and $A=6$ creates two classes of traffic which have different probabilities of success. However, even when the carrier sense range is large, 
the difference in success probabilities remains modest: around $15 \%$.

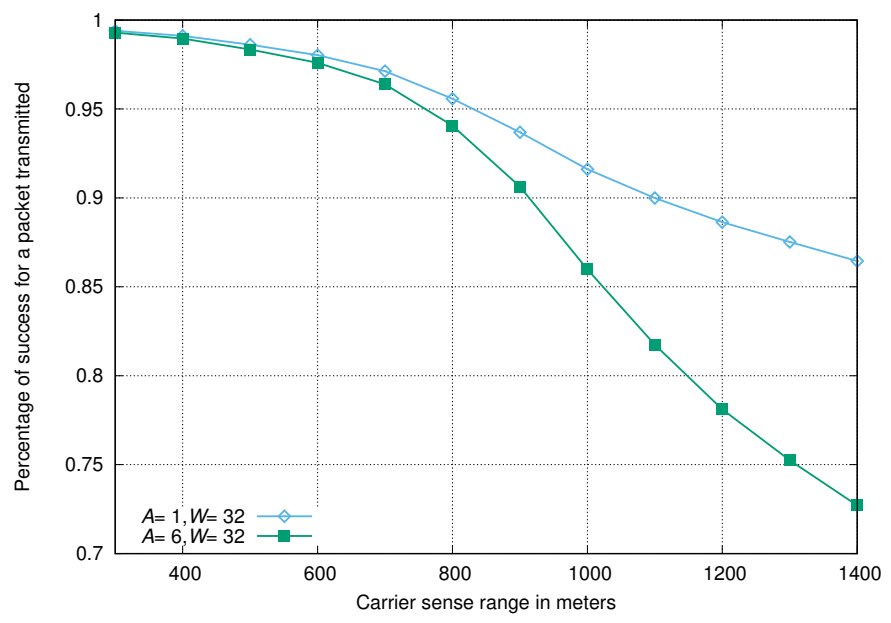

Fig. IV.1. Percentage of successful transmissions for both kinds of traffic with different AIFS values.

In Figure IV.2, we show the actual throughput for the two classes of traffic with the EDCF scheme with $A=1$ and $A=6$. In contrast to what we note for the probability of success, the EDCF scheme creates two very different classes of traffic. When the carrier sense range increases above 900 m the class of traffic with $A=1$ continues to increase its throughput whereas the class of traffic with $A=6$ starts to decrease its throughput. The scheme operates as if the low priority traffic vanishes in order to leave room for the high priority traffic.

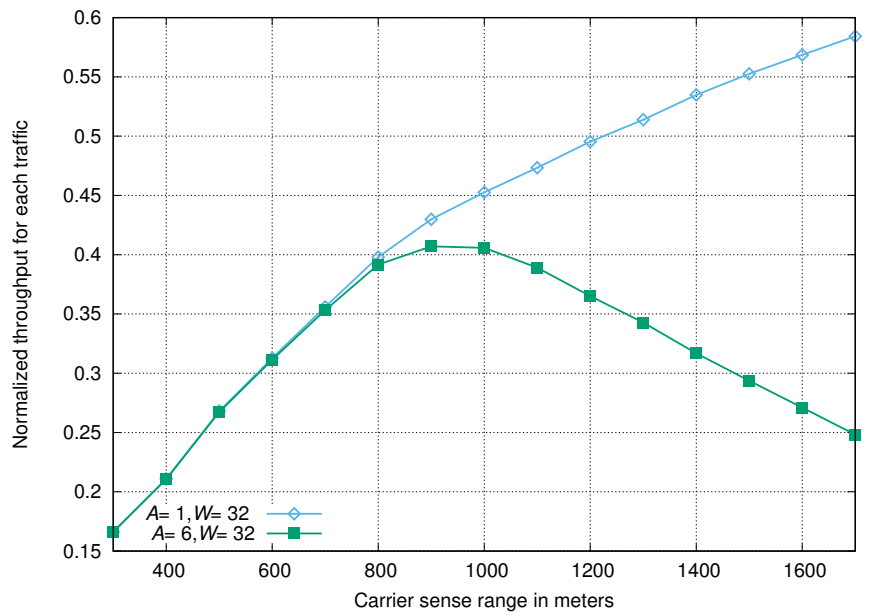

Fig. IV.2. Throughput for both kinds of traffic with different AIFS values.

In Figure IV.3, we study the effect of varying the values of $A$ on the performance. We study three cases: two classes of traffic with $A=1$ and $A=6$, two classes of traffic with $A=1$ and $A=8$ and finally two classes of traffic with $A=1$ and $A=10$. Surprisingly, we observe that increasing the difference between the two values of $A$ actually reduces the difference between the success rates. Moreover the average success rate is larger when the difference between the two values of $A$ increases.

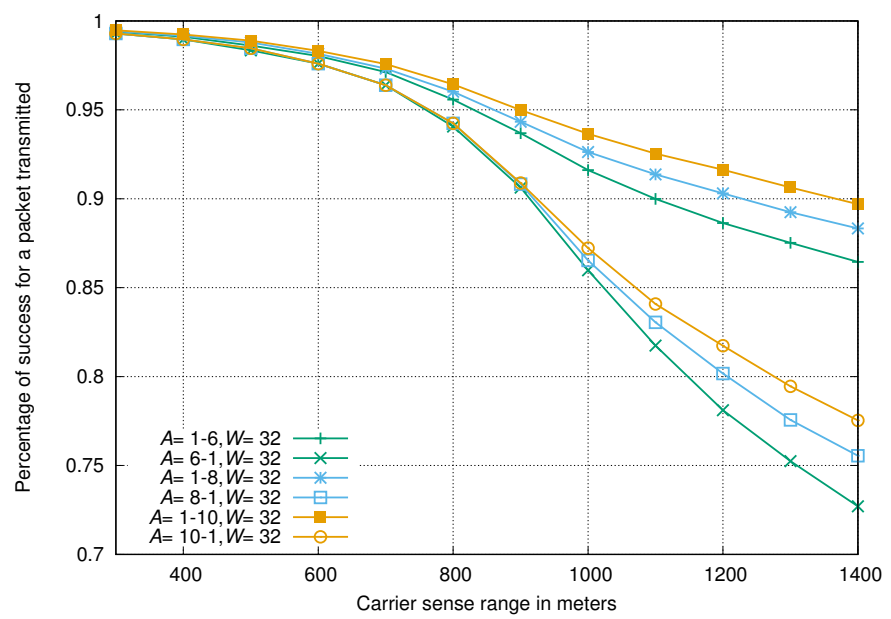

Fig. IV.3. Percentage of successful transmissions for both kinds of traffic with different AIFS values. Effect of different values for $A$

In Figure IV.4, we study the effect of varying the values of $A$ on the throughput. We study three cases: two classes of traffic with $A=1$ and $A=6$, two classes of traffic with $A=1$ and $A=8$ and finally two classes of traffic with $A=1$ and $A=10$. This time, the result can be predicted, when the difference between the two values of $A$ increases, the difference between the throughput of the two traffics also tends to increase.

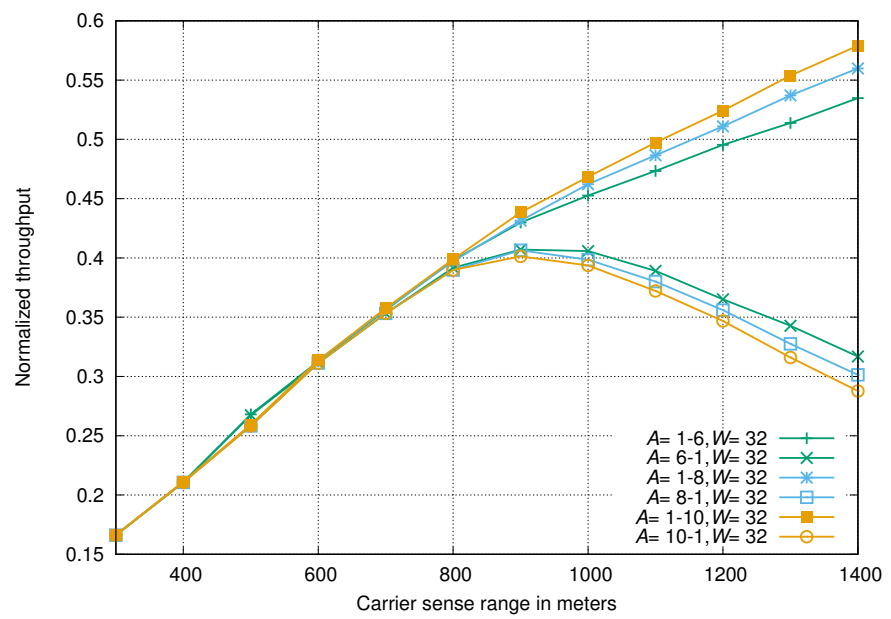

Fig. IV.4. Throughput for both kinds of traffic with different AIFS values. Effect of different values for $A$

In Figure IV.5, we compare the effect of varying the values of $A$ and varying the values of $W$ on the percentage of successful transmission. We set up a network with two types of vehicles, one using $A=1$ and the other $A=6$, both with $W=32$ and another network with two types of vehicles, one using $W=8$ and the other $W=64$, both with $A=1$. We observe that the differentiation obtained by varying the values of $A$ is much greater than that obtained by varying the values of $W$. 


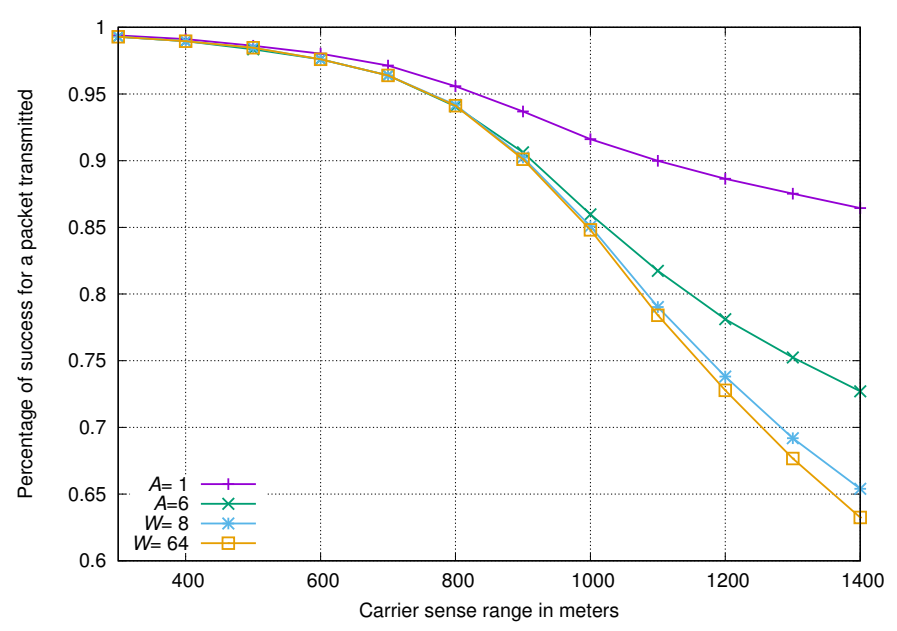

Fig. IV.5. Percentage of successful transmissions for both kinds of traffic. Comparison between varying $A$ and varying $W$

In Figure IV.6, we compare the effect of varying the values of $A$ and varying the values of $W$ on normalized throughput. We compare a network with two types of vehicles, one using $A=1$ and the other $A=6$, both with $W=32$ and another network with two types of vehicles, one using $W=8$ and the other $W=64$, both with $A=1$. We observe that the differentiation obtained by varying the values of $A$ is much greater than that obtained by varying the values of $W$.

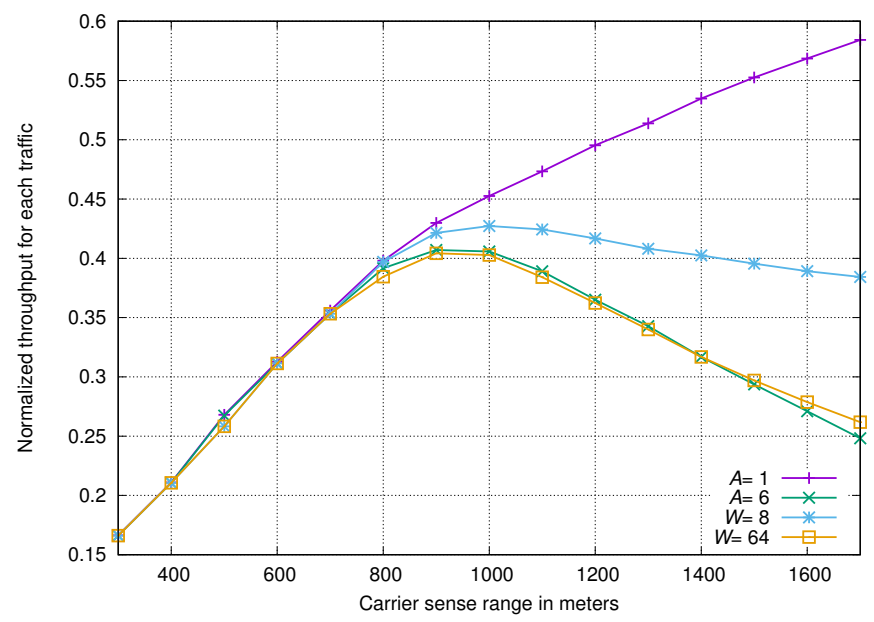

Fig. IV.6. Throughput for both kinds of traffic with different AIFS values. Comparison between varying $A$ and varying $W$

\section{CONCLUSION}

In this paper, we present a simple probabilistic model for the IEEE 802.11 Enhanced Distributed Coordination Function (EDCF) which can build different access priorities for different classes of traffic. These priorities are obtained using different inter frame spacings called Arbitration Inter Frame Spacings (AIFSs) to differentiate the access of different classes of traffic. The model is composed of Markov chains (node Markov chains) which, for each class of traffic, provide the back-off timer and possibly the position in the inter frame spacing and a Markov chain (the back-off Markov chain) which provides the current value of the back-off timer during the back-off process. To assess the performance of the network, one must solve the coupled Markov chains in order to obtain the busy rate ratio in each class of traffic. These solutions must be used in the steady-state of the back-off Markov chain to be able to derive the success probability and the throughput rate of each traffic class.

The results provided by the analytical model show that using different value of $A$ to differentiate two traffic classes actually has a very significant impact on the throughput when the input load increases, whereas the impact on the probability of successful transmission remains moderate. The model shows that a small difference (e.g. $A=1$ and $A=6$ ) between the two values of $A$ is enough to obtain a very significant effect on the throughput.

When we compare the effect obtained by varying $A$ and the effect obtained by varying $W$ we note that varying $A$ is much more efficient to differentiate the performance of traffics sharing the same radio spectrum.

\section{REFERENCES}

[1] B. B. Zhenning Kong, Danny H. K. Tsang and D. Gao, "Performance analysis of IEEE $802.11 \mathrm{e}$ contention-based channel access," IEEE Journal on Selected Areas in Communications, vol. 22, no. 10, pp. 2095-2106, 2004.

[2] L. Xiong and G. Mao, "Saturated throughput analysis of ieee $802.11 \mathrm{e}$ edca," Computer Networks, vol. 51, no. 11, pp. 3047-3068, 2007.

[3] H. Wu, X. Wang, Q. Zhang, and X. Shen, "IEEE 802.11 e enhanced distributed channel access (EDCA) throughput analysis," in Communications, 2006. ICC'06. IEEE International Conference on, vol. 1. IEEE, 2006, pp. 223-228.

[4] Task Group p. IEEE 802.11p, Wireless Access in Vehicular Environments (WAVE) Draft Standard, 2007.

[5] ETSI EN 302 637-2, "Intelligent Transport Systems (ITS) - Vehicular Communications - Basic Set of Applications - Part 2 : Specification of Cooperative Awareness Basic Service," History, vol. 1, pp. 1-44, 2014.

[6] ETSI EN 302 637-3, "Intelligent Transport Systems (ITS); Vehicular Communications; Basic Set of Applications; Part 3: Specifications of Decentralized Environmental Notification Basic Service," vol. 2, pp. 1-73, 2014.

[7] L. Kleinrock and F. Tobagi, "Packet switching in radio channels: Part I-carrier sense multiple-access modes and their throughput-delay characteristics," IEEE Transactions on Communications, vol. COM23, no. 12, pp. 1400-1416, December 1975, (Also, "Multiple Access Communications, Foundations for Emerging Technologies", Norman Abramson (Ed), IEEE Press, 1992, pp. 272-288.).

[8] G. Bianchi, "Performance Analysis of the IEEE 802.11 Distributed Coordination Function," IEEE Journal of Selected Areas in Communications., vol. 18, no. 3, pp. 535-547, March 2000. [Online]. Available: http://dx.doi.org/10.1109/49.840210

[9] P. Muhlethaler and A. Najid, "Throughput optimization in multihop csma mobile ad hoc networks," in EW 2004. The 5th European Wireless Conference, February 24 - 27. Barcelona 2004.

[10] D. Stoyan, W. S. Kendall, and J. Mecke, Stochastic geometry and its applications. 2nd edition. Wiley, 1995.

[11] A. Busson and G. Chelius, "Point processes for interference modeling in csma/ca ad-hoc networks," in Conference: Proceedings of the 6th ACM International Workshop on Performance Evaluation of Wireless Ad Hoc, Sensor, and Ubiquitous Networks, PE-WASUN 2009, October 28-29 2009, Tenerife, Canary Islands, Spain, 2009.

[12] C. Xu and Z. Yang, "Non-saturated throughput analysis of ieee 802.11 ad hoc networks," IEICE - Trans. Inf. Syst., vol. E89-D, no. 5, pp. 1676-1678, May 2006. [Online]. Available: http://dx.doi.org/10.1093/ietisy/e89-d.5.1676 